Supporting Information for:

\title{
Cellular Engineering with Membrane Fusogenic Liposomes to Produce Functionalized Extracellular Vesicles
}

Junsung Lee, ${ }^{\mathrm{a}, \mathrm{b}, \mathrm{c}, 1}$ Hyoungjin Lee, ${ }^{\mathrm{a}, \mathrm{c}, 1}$ Unbyeol Goh, ${ }^{\mathrm{a}, \mathrm{c}, 1}$ Jiyoung Kim, ${ }^{\mathrm{a}, \mathrm{c}}$ Moonkyoung Jeong, ${ }^{\mathrm{a}, \mathrm{c}}$ Jean Lee, ${ }^{\mathrm{a}, \mathrm{c}}$ and Ji-Ho Park ${ }^{\mathrm{a}, \mathrm{b}, \mathrm{c}, \mathrm{d}, *}$

${ }^{\mathrm{a}}$ Department of Bio and Brain Engineering, ${ }^{\mathrm{b}}$ Graduate School of Medical Science and Engineering, ${ }^{\mathrm{c}}$ Institute for Health Science and Technology, and ${ }^{\mathrm{d}}$ Institute for the Nanocentury, Korea Advanced Institute of Science and Technology (KAIST), Daejeon, 34141, Republic of Korea.

${ }^{1}$ These authors contributed equally to this work.

*Address correspondence to jihopark@kaist.ac.kr 


\section{EXPERIMENTAL METHODS}

Cell culture. MDA-MB-231 human breast cancer cells were cultured in RPMI (Hyclone, USA) containing 10\% fetal bovine serum (FBS, Hyclone) and 1\% antibiotics, penicillin and streptomicyn (Hyclone). B16F10 mouse melanoma cells were cultured in DMEM (Hyclone), containing 10\% fetal bovine serum (FBS, Hyclone) and 1\% penicillin/streptomycin (Hyclone). All cells were incubated at $37^{\circ} \mathrm{C}$ in $5 \% \mathrm{CO}_{2}$.

Liposome preparation and characterization. Various liposomal formulations were prepared from 1,2-dimyristoyl-sn-glycero-3-phosphocholine (DMPC, Avanti Polar Lipids), hydrogenated soy L- $\alpha$-phosphatidylcholin (HSPC, Avanti Polar Lipids), 1,2-dimyristoyl-sn-glycero-3phosphocholine (DMPC, Avanti Polar Lipids), or 1,2-dipalmitoyl-sn-glycero-3-phosphocholine (DPPC, Avanti Polar Lipids), 1,2-diphytanoyl-sn-glycero-3-phosphoethanolamine (DPhPE, Avanti Polar Lipids), 1,2-distearoyl-sn-glycero-3-phosphoethanolamine-N[methoxy(polyethylene glycol)-2000] (DSPE-PEG), and 1,2-dioleoyl-3-trimethylammoniumpropane (DOTAP) using a film hydration/extrusion method. Membrane fusogenicity of liposomes on cancer cells was optimized by adjusting the molar ratios of DMPC, DSPE-PEG, and DOTAP. Membrane fusogenic liposomes (MFL) and non-fusogenic liposomes (NFL) were prepared from DMPC, DSPE-PEG, and DOTAP at 76.15:3.85:20 and 80:0:20 molar ratios, respectively. For liposomes loaded with hydrophilic cargo, carboxyfluorescein (CF, Sigma) was incorporated into the internal space of the liposomes using a hydration protocol. The lipid film was prepared with 725.5 $\mu \mathrm{g}$ DMPC, $151.6 \mu \mathrm{g}$ DSPE-PEG, and 196.3 $\mu \mathrm{g}$ DOTAP (for MFL) or with $761.7 \mu \mathrm{g}$ DMPC and 196.3 $\mu \mathrm{g}$ DOTAP (for NFL) by evaporating the organic solvent. The film was then hydrated with $1 \mathrm{~mL}$ CF solution (80 $\mathrm{mM}$ in PBS). After hydration and membrane (100 nm) extrusion, free CF was removed by ultracentrifugation (Hanil Science, Korea) or 
Sephadex G-50 column (GE healthcare). For liposomes loaded with lipophilic cargo, 1,1'dioctadecyl-3,3,3',3'-tetramethylindocarbocyanine perchlorate (DiI, Invitrogen) or 1,1'dioctadecyl-3,3,3',3'-tetramethylindodicarbocyanine perchlorate (DiD, Invitrogen) was incorporated into the liposomal membrane using a hydration protocol. The DiI or DiDincorporated lipid film was prepared with $725.5 \mu \mathrm{g}$ DMPC, $151.6 \mu \mathrm{g}$ DSPE-PEG, and $196.3 \mu \mathrm{g}$ DOTAP (for MFL) or with $761.7 \mu \mathrm{g}$ DMPC and $196.3 \mu \mathrm{g}$ DOTAP (for NFL), and $26.3 \mu \mathrm{g}$ DiI or DiD by evaporating the organic solvent. The film was then hydrated with $1 \mathrm{~mL}$ PBS. After hydration and membrane (100 nm) extrusion, free DiI or DiD was removed by ultracentrifugation or Sephadex G-50 column. For liposomes loaded with therapeutic cargo, hydrophilic tirapazamine (TPZ, Sigma) or hydrophobic paclitaxel (PTX, Sigma) were incorporated into the internal space or the membrane of the liposomes using a hydration protocol, respectively. For liposomes loaded with hydrophobic drug, the lipid film was prepared with $725.5 \mu \mathrm{g}$ DMPC, 151.6 $\mu \mathrm{g}$ DSPE-PEG, and $196.3 \mu \mathrm{g}$ DOTAP (for MFL) or with $761.7 \mu \mathrm{g}$ DMPC and $196.3 \mu \mathrm{g}$ DOTAP (for NFL), and $12 \mu \mathrm{g}$ PTX (Sigma) by evaporating the organic solvent. The film was then hydrated with $1 \mathrm{~mL}$ PBS. After hydration and membrane (100 nm) extrusion, free PTX was removed by ultracentrifugation or Sephadex G-50 column. For liposomes loaded with hydrophilic drug, the lipid film was prepared with $725.5 \mu \mathrm{g}$ DMPC, 151.6 $\mu \mathrm{g}$ DSPE-PEG, and 196.3 $\mu$ g DOTAP (for MFL) or with $761.7 \mu \mathrm{g}$ DMPC and 196.3 $\mu \mathrm{g}$ DOTAP (for NFL) by evaporating the organic solvent. The film was then hydrated with $1 \mathrm{~mL}$ of PBS containing $1.4 \mathrm{mM}$ of TPZ. After hydration and membrane (100 nm) extrusion, free TPZ was removed by ultracentrifugation or Sephadex G-50 column. For liposomes incorporated with functional lipids, the 5\% molar ratio of DMPC were substituted with functional lipids during the lipid film preparation. Functional lipids used in this study include 1,2-dimyristoyl-sn-glycero-3- 
phosphoethanolamine-N-(7-nitro-2-1,3-benzoxadiazol-4-yl) (ammonium salt) (14:0 NBD-PE, Avanti Polar Lipids), 1,2-dipalmitoyl-sn-glycero-3-phosphoethanolamine-N-(7-nitro-2-1,3benzoxadiazol-4-yl) (ammonium salt) (16:0 NBD-PE, Avanti Polar Lipids), 1,2-diphytanoyl-snglycero-3-phosphoethanolamine-N-(7-nitro-2-1,3-benzoxadiazol-4-yl) (ammonium salt) (16:0 NBD-DPhPE, Avanti Polar Lipids), 1,2-distearoyl-sn-glycero-3-phosphoethanolamine-N-(7nitro-2-1,3-benzoxadiazol-4-yl) (ammonium salt) (18:0 NBD-PE, Avanti Polar Lipids), 1,2dioleoyl-sn-glycero-3-phospho-L-serine-N-(7-nitro-2-1,3-benzoxadiazol-4-yl) (ammonium salt) (18:1 NBD-PS, Avanti Polar Lipids), 1-palmitoyl-2-\{12-[(7-nitro-2-1,3-benzoxadiazol-4yl)amino]dodecanoyl \} 1-sn-glycero-3-phosphoethanolamine (16:0-12:0 NBD-PE, Avanti Polar Lipids), and 1,2-diphytanoyl-sn-glycero-3-phosphoethanolamine (4ME 16:0 PE, Avanti Polar Lipids). For liposomes incorporated with azide-lipids (Azide-MFLs or -NFLs), liposomes containing DPhPE at the molar ratio of 5\% were prepared and then reacted with azidoacetic acid NHS ester (Click Chemistry Tools, USA) for $2 \mathrm{~h}$ as a molar ratio of 1:10. Unconjugated compounds were removed by using Sephadex G-50 Medium or centrifugal filter (100K MWCO, Millipore, USA). The hydrodynamic size and zeta potential of the liposomes were measured using dynamic light scattering (Zetasizer Nano ZS90, Malvern Instruments). The absorbance and fluorescence of the cargo-loaded liposomes were measured using UV-Vis spectrophotometer and spectrofluorometer (Molecular Devices), respectively ( $\lambda_{\mathrm{ex}}=630 \mathrm{~nm}$ and $\lambda_{\mathrm{em}}=670 \mathrm{~nm}$ for DiD; $\lambda_{\mathrm{ex}}=490 \mathrm{~nm}$ and $\lambda_{\mathrm{em}}=520 \mathrm{~nm}$ for CF, CF, and NDB; $\lambda_{\mathrm{ex}}=530 \mathrm{~nm}$ and $\lambda_{\mathrm{em}}=570 \mathrm{~nm}$ for Dil in the fluorescence measurements). The cytotoxicity of the liposomes was evaluated using MTT assay. $1 \times 10^{4}$ of cancer cells seeded in 96-well tissue culture plates (SPL Life Science, USA) were treated with synthetic liposomes at various lipid concentrations for $30 \mathrm{~min}$ and further incubated for $48 \mathrm{~h}$. The cells were then incubated with thiazolyl blue tetrazolium bromide 
solution (MTT, Sigma) for $4 \mathrm{~h}$, and the supernatant was removed. The formed violet crystals were dissolved in DMSO (Sigma) and the absorbance at $570 \mathrm{~nm}$ was measured using the UV-Vis spectrophotometer. For fusion assay, liposomes loaded with a self-quenching lipid dye octadecyl rhodamine B (R18, Invitrogen) were prepared as described previously ${ }^{21}$. The amount of R18 loaded onto liposome membranes was $10 \%$ of the total lipid amount. $1 \times 10^{4}$ of cancer cells were seeded into black 96-well tissue culture plates (SPL Life Science, USA) and then treated with R18-loaded liposomes at a lipid concentration of $5.62 \mu \mathrm{M}$. The fluorescence dequenching of R18 was measured at $37^{\circ} \mathrm{C}$ every $5 \mathrm{~min}$ over a 30 -min period using the spectrofluorometer $\left(\lambda_{\mathrm{ex}}=550\right.$ $\mathrm{nm}$ and $\lambda_{\mathrm{em}}=590 \mathrm{~nm}$ ). The percentage of fluorescence dequenching was calculated according to the following equation ${ }^{21}$; Fluorescence dequenching $(\mathrm{FD}, \%)=\frac{\left(F-F_{i}\right)}{F_{\max }-F_{i}} \times 100$, where $F$ is the fluorescence intensity of cells treated with R18-loaded liposomes at specific time points, $F_{i}$ is the fluorescence intensity of the cells treated with R18-loaded liposomes for less than $30 \mathrm{~s}$, and $F_{\max }$ is the fluorescence intensity of the cells disrupted with Triton X-100 (Sigma) after a 30-min treatment with R18-loaded liposomes.

In vitro fluorescence cellular imaging. To observe membrane fusogenicity of liposomes and their intracellular cargo delivery, $2 \times 10^{4}$ of cells were treated with liposomes loaded with fluorescent cargo (DiD, CF, or NBD-lipids) for $30 \mathrm{~min}$ at a lipid concentration of $281 \mu \mathrm{M}$. The cells were washed with PBS three times, stained with Hoechst 33342 (Invitrogen), and imaged using confocal microscopy (Nikon Instrument Inc). To observe membrane fusogenicity of liposomes containing azide-lipids, $2 \times 10^{4}$ of cells were treated with liposomes containing azidelipids for $30 \mathrm{~min}$ at a lipid concentration of $281 \mu \mathrm{M}$, and washed with PBS three times. The cells incorporated with azide-lipids were then reacted with DBCO-carboxyrhodamine 110 (BDCO- 
CR, Click Chemistry Tools, USA) for 20 min at a concentration of $10 \mu \mathrm{M}$, washed with PBS three times, and imaged using confocal microscopy.

Isolation and characterization of functionalized EVs. All cancer cells were cultured in the conditioned media with extracellular vesicle (EV)-depleted FBS. $1.2 \times 10^{7}$ of cells were treated with free cargo or cargo-loaded liposomes for $30 \mathrm{~min}$ at a lipid concentration of $281 \mu \mathrm{M}$, and then washed to remove free cargo or liposomes in the media. The supernatants were collected from the treated cells $48 \mathrm{~h}$ after incubation. The cells and debris were removed by a series of centrifugations at $300 \mathrm{~g}$ for $5 \mathrm{~min}, 2000 \mathrm{~g}$ for $20 \mathrm{~min}$, and $10,000 \mathrm{~g}$ for $30 \mathrm{~min}$ at $4^{\circ} \mathrm{C}$. Cargoloaded EVs were isolated from the final supernatant sample by ultracentrifugation at $110,000 \mathrm{~g}$ for $120 \mathrm{~min}$ at $4^{\circ} \mathrm{C}$. The EV pellet was washed twice and resuspended in PBS. The amount of cargo-loaded EVs produced from the treated cells was quantified using a BCA protein assay kit (Thermo Scientific). To investigate the changes of the amount of cargo loaded in EVs after treatment of various concentrations of MFLs to parental cells, the cells were treated with 0,35 , $70,140,281,562,1124$, or $2248 \mu \mathrm{M}$ of MFLs containing NBD-DPhPE for $30 \mathrm{~min}$. The fluorescence intensities of the cells immediately after MFL treatment and the EVs isolated at 48 $\mathrm{h}$ after MFL treatment were measured to quantify the cellular uptake of MFLs and the cargo encapsulation in the EVs, respectively, using the spectrofluorometer. The hydrodynamic size and zeta potential of control and cargo-loaded EVs were measured using dynamic light scattering. The morphology of control and cargo-loaded EVs were observed using transmission electron microscopy (TEM, 300KV, Hitachi). A drop of the solution of EVs was put on a formvar/carbon coated grid (Ted Pella, Inc,) and dried. EVs-coated grid was stained with 2\% phosphotungstic acid ( $\mathrm{pH} 8.0$, Sigma) for $1 \mathrm{~min}$ before visualization with TEM. The absorbance and fluorescence of cargo-loaded EVs were measured using the UV-Vis spectrophotometer and the 
spectrofluorometer, respectively. The protein profile of control and cargo-loaded EVs was visualized on the polyacrylamide gel using Coomassie blue staining according to the manufacture's protocol (Bio-rad). The incorporation efficiency of fluorescent cargo in the EVs was calculated with the following equation; Incorporation efficiency $(\%)=F_{\mathrm{EV}} / F_{\text {cell }} \times 100$, where $F_{\text {cell }}$ is the fluorescence intensity of the cells treated with cargo-loaded liposomes for 30 $\min$, and $F_{\mathrm{EV}}$ is the fluorescence intensity of the EVs $(250 \mu \mathrm{g})$ produced from the liposometreated cells for $48 \mathrm{~h}$. The fluorescence intensities of EVs and cells were measured using the spectrofluorometer after disrupting the membrane with RIPA buffer (Sigma) to avoid the fluorescence quenching. To verify the presence of azide-lipids on the EVs produced from the cells treated with azide-MFLs, $250 \mu \mathrm{g}$ of EVs were reacted with $4 \mu \mathrm{g}$ of DBCO-CR for $30 \mathrm{~min}$. Unconjugated DBCO-CR was removed with the Sephadex G-50 column. The fluorescence intensity of the EVs was then measured using the spectrofluorometer.

Transwell experiments. Cancer cell monolayers were grown on a polycarbonate filter $(0.4 \mu \mathrm{m}$ pore) of the upper chamber of a transwell (SPL Lifescience). For fluorescence experiments, the cells on the transwell filter were treated with DiD-loaded liposomes $(281 \mu \mathrm{M})$ for $30 \mathrm{~min}$ and washed to remove free liposomes in the media. The treated cells on the transwell filter were then placed on the lower chamber onto which fresh cancer cells were plated, and incubated for $48 \mathrm{~h}$ to allow production of DiD-loaded EVs and their subsequent internalization into the cells in the lower chamber. To visualize cellular uptake of EVs, the cells in the lower chambers were stained with Hoechst 33342 and imaged using confocal microscopy. For fluorescence quantification, the fluorescence intensity of the cells in the lower chambers was measured using the spectrofluorometer after lysing with RIPA buffer. For therapeutic experiments with PTX, the cells on the transwell filter were treated with PTX-loaded liposomes at a PTX concentration of 
$600 \mathrm{nM}$ for $30 \mathrm{~min}$ and washed to remove free liposomes in the media. The treated cells on the transwell filter were then placed on the lower chamber onto which fresh cancer cells were plated, and incubated for $48 \mathrm{~h}$ to allow production of PTX-loaded EVs and their subsequent internalization into the cells in the lower chamber. The cell viability in both upper filter and lower chamber was then evaluated using MTT assay. For therapeutic experiments with TPZ, the cells on the transwell filter were treated with TPZ-loaded liposomes at a TPZ concentration of 36 $\mu \mathrm{M}$ for $30 \mathrm{~min}$ and washed to remove free liposomes in the media. The treated cells on the transwell filter were then placed on the lower chamber onto which fresh cancer cells were plated, and incubated for $48 \mathrm{~h}$ to allow production of TPZ-loaded EVs and their subsequent internalization into the cells in the lower chamber. The cells were further incubated in hypoxic $\left(1 \% \mathrm{O}_{2}, 5 \% \mathrm{CO}_{2}\right)$ condition for $24 \mathrm{~h}$ to induce hypoxia-specific cytotoxicity. The cell viability in both upper filter and lower chamber was then evaluated using MTT assay. For targeting experiments with clickable EVs, DBCO-CGKRK was prepared by reacting $2.4 \mathrm{mg}$ of CGKRK (in $1 \mathrm{ml}$ of PBS) with $1.2 \mathrm{mg}$ of DBCO-maleimide (in $100 \mu \mathrm{l}$ of DMSO, Click Chemistry Tools, USA). B16F10 cells on the transwell upper filter were treated with liposomes co-loaded with azide-lipids and DiD or PTX for $30 \mathrm{~min}$ and washed to remove free liposomes in the media. The treated cells on the upper filter were then placed on the lower chamber onto which fresh B16F 10 cells were plated, and incubated in the medium containing DBCO-CGKRK solution $(25: 1, \mathrm{v} / \mathrm{v})$ for $48 \mathrm{~h}$ to allow production of azide-EVs loaded with DiD or PTX, and their subsequent click conjugation with DBCO-CGKRK. The CGKRK-EVs loaded with DiD or PTX were finally internalized into cells in the lower chamber. For EV-depleted supernatant treatments, the cells were treated with drug-loaded liposomes $(281 \mu \mathrm{M})$ for $30 \mathrm{~min}$, washed to remove free liposomes in the media, and further incubated for $48 \mathrm{~h}$. The drug-loaded EVs secreted from the cells were 
removed from the supernatant of the treated cells by ultracentrifugation. Fresh cancer cells were incubated with the EV-depleted supernatant for $24 \mathrm{~h}$, and the cell viability was evaluated using MTT assay.

Statistics. Statistical analysis of the data was performed using one-way or two-way analysis of variance (ANOVA) or unpaired t-test followed by a Bonferroni post test. All experiments were performed at least three times. 


\begin{tabular}{|c|c|c|c|c|c|c|c|c|c|}
\hline \multirow{2}{*}{ Liposome } & \multicolumn{6}{|c|}{ Lipid composition (mol\%) } & \multirow{2}{*}{$\begin{array}{l}\text { Size } \\
(\mathrm{nm})\end{array}$} & \multirow{2}{*}{$\begin{array}{c}\text { Surface } \\
\text { Charge } \\
(\mathrm{mV})\end{array}$} & \multirow{2}{*}{ Note } \\
\hline & DOPC & DMPC & DPPC & HSPC & PEG-PE & DOTAP & & & \\
\hline L1 & - & 56.15 & - & - & 3.85 & 40 & 120.0 & 29.0 & \\
\hline L2 & - & 76.15 & - & - & 3.85 & 20 & 115.7 & 17.5 & MFL \\
\hline L3 & - & 86.15 & - & - & 3.85 & 10 & 117.3 & 6.0 & \\
\hline L4 & & 96.15 & & & 3.85 & - & 116.3 & -13.4 & \\
\hline
\end{tabular}
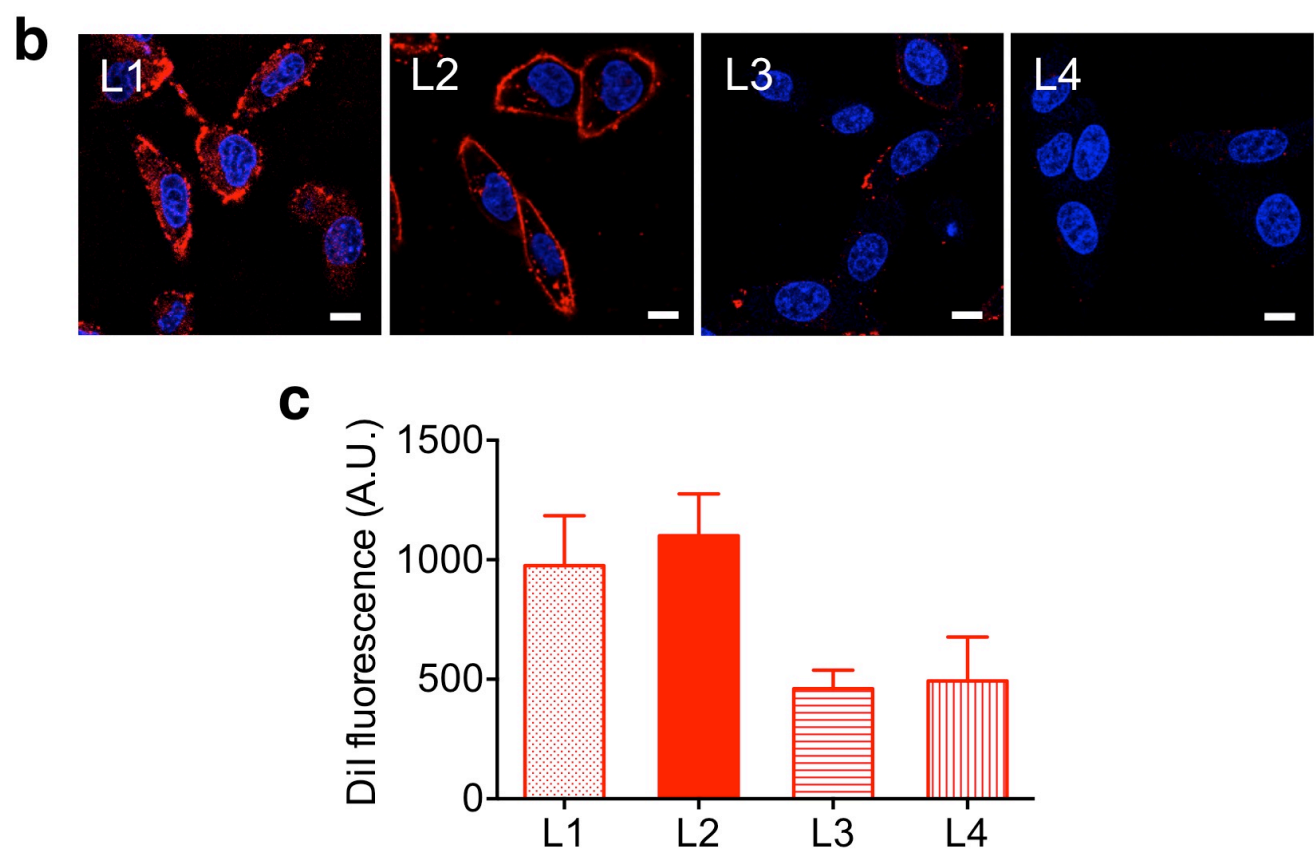

Figure S1 Membrane fusogenicity of liposome formulations composed with DMPC, PEG-PE and DOTAP and their incorporation efficiency of cargo in extracellular vesicles (EVs). (a) Lipid composition and physicochemical properties of liposome formulations composed with DMPC, PEG-PE and DOTAP. Mean hydrodynamic sizes of the liposomes were measured based on dynamic light scattering measurements $(n=3)$. Mean surface charges of the liposomes were measured based on zeta zeta-potential measurements $(n=3)$. (b) Confocal microscopic images of B16F10 cells treated with liposomes loaded with lipophilic cargo DiI (red) for $30 \mathrm{~min}$. Nuclei were stained with Hoechst (blue). Scale bar indicates $5 \mu \mathrm{m}$. (c) Fluorescence quantification of DiI in equal quantities of EVs secreted from cells treated with liposomes loaded with DiI. MFL denotes membrane fusogenic liposomes. Data represent averages \pm S.D. 
a

\begin{tabular}{|c|c|c|c|c|c|c|c|c|c|}
\hline & \multicolumn{5}{|c|}{ Lipid composition (mol\%) } & \multicolumn{2}{|c|}{$\begin{array}{c}\text { Size } \\
\text { Liposome }\end{array}$} & $\begin{array}{c}\text { Surface } \\
\text { Charge } \\
(\mathrm{mm})\end{array}$ & Note \\
\cline { 2 - 12 } & DOPC & DMPC & DPPC & HSPC & PEG-PE & DOTAP & & MFL \\
\hline L2 & - & 76.15 & - & - & 3.85 & 20 & 115.7 & 17.5 & MF \\
\hline L5 & 76.15 & - & - & - & 3.85 & 20 & 117.4 & 21.2 & \\
\hline L6 & - & - & 76.15 & - & 3.85 & 20 & 133.4 & 19.5 \\
\hline L7 & - & - & - & 76.15 & 3.85 & 20 & 120.4 & 15.5 \\
\hline
\end{tabular}

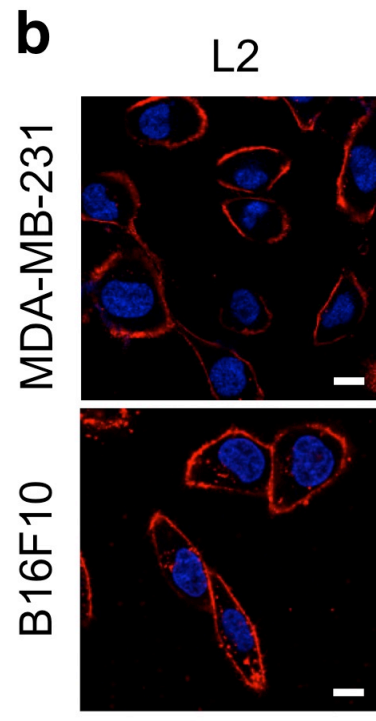

L5

L6

L7
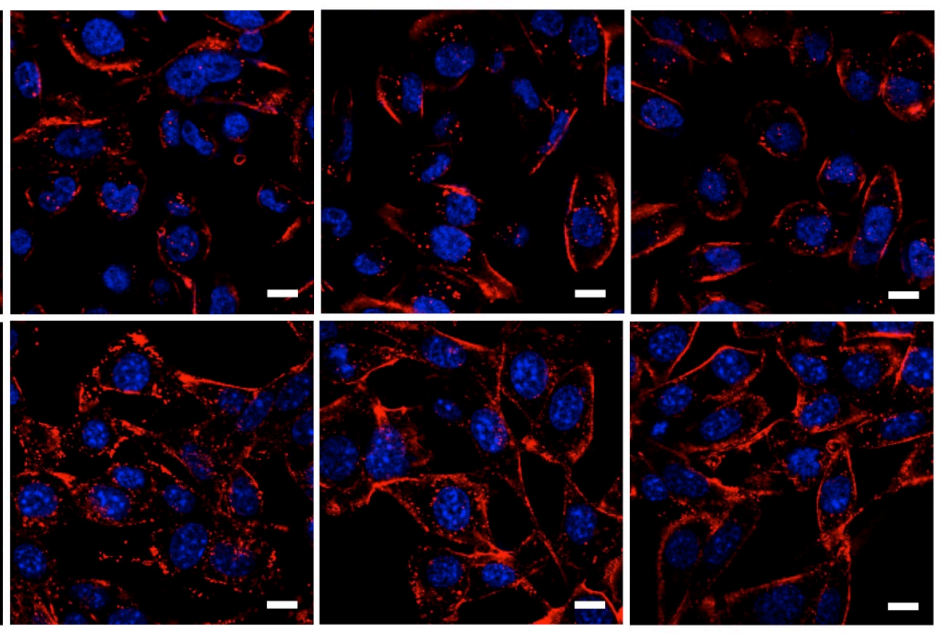

C

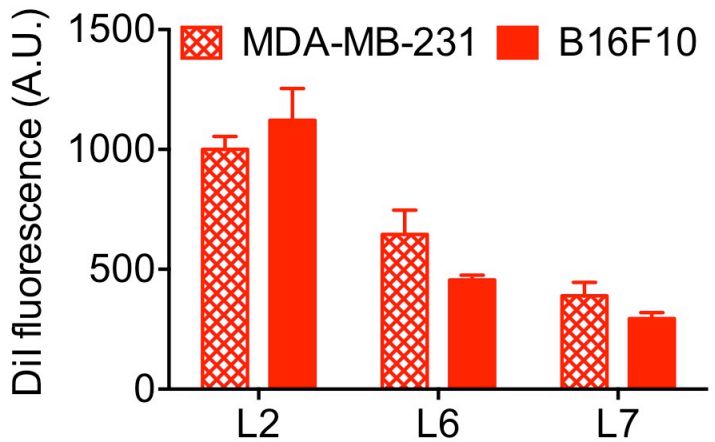

Figure S2 Membrane fusogenicity of liposome formulations composed with different base lipids and their incorporation efficiency of cargo in EVs. (a) Lipid composition and physicochemical properties of liposome formulations composed with different base lipids. Mean hydrodynamic sizes of the liposomes were measured based on dynamic light scattering measurements $(n=3)$. Mean surface charges of the liposomes were measured based on zeta zeta-potential measurements $(\mathrm{n}=3)$. (b) Confocal microscopic images of B16F10 cells treated with liposomes loaded with lipophilic cargo DiI (red) for 30 min. Nuclei were stained with Hoechst (blue). Scale bar indicates $5 \mu \mathrm{m}$. (c) Fluorescence quantification of DiI in equal quantities of EVs secreted from cells treated with liposomes loaded with DiI. Data represent averages \pm S.D. 

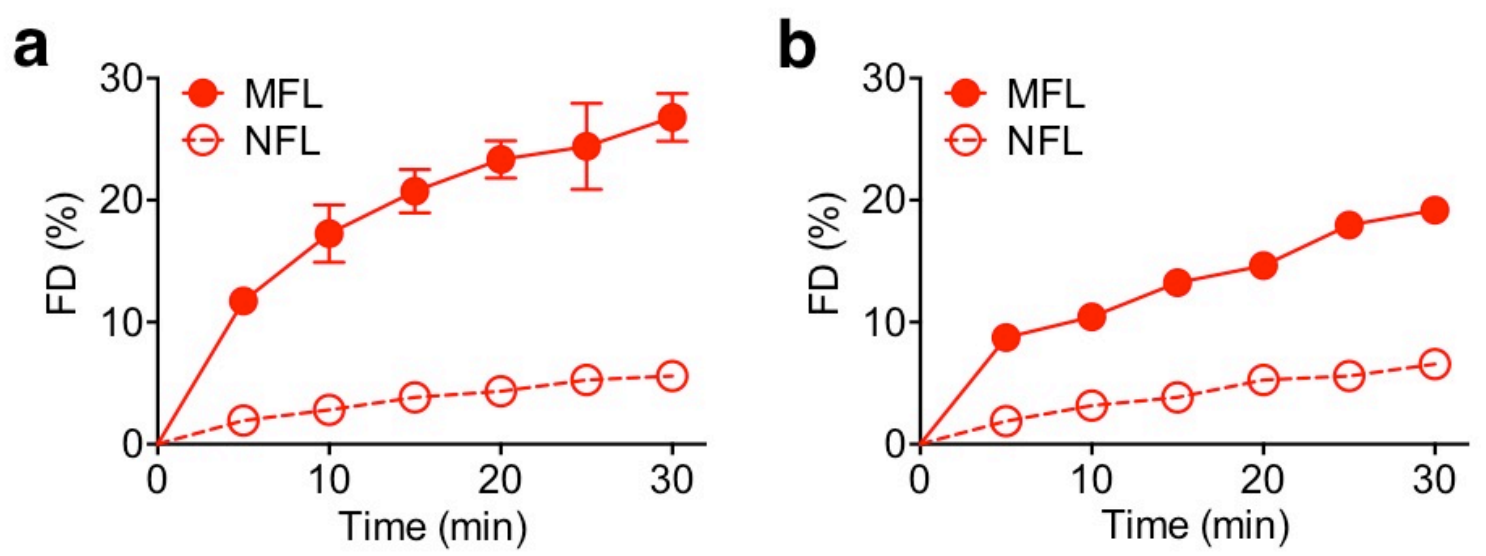

Figure S3 Membrane fusogenicity of MFLs and NFLs on cancer cells. (a and $\mathbf{b}$ ) Membrane fusogenicity of synthetic liposomes based on fluorescence dequenching (FD) of MDA-MB-231 (a) and B16F10 cells (b) treated with liposomes loaded with self-quenching hydrophobic dye R18 over a 30-min period. Results are presented as a percentage of R18 fluorescence dequenching over the maximum fluorescence intensity of R18 added to the cells. Data represent averages \pm S.D. 


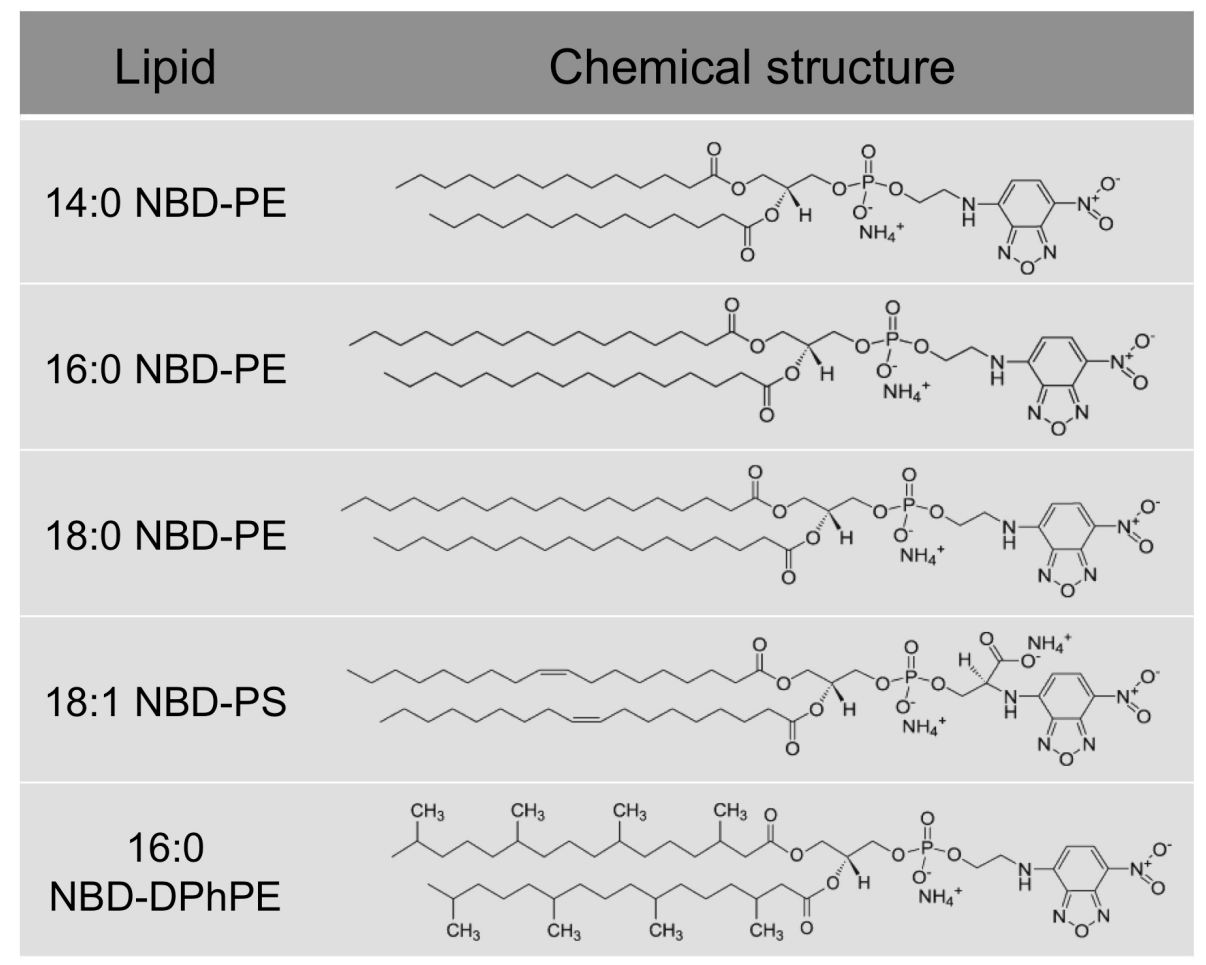

PE: Phosphoethanolamine, PS: Phosphatidylserine, NBD: hydrophilic dye

Figure S4 Types and chemical structures of fluorescent phospholipids used in this study to engineer EVs. 


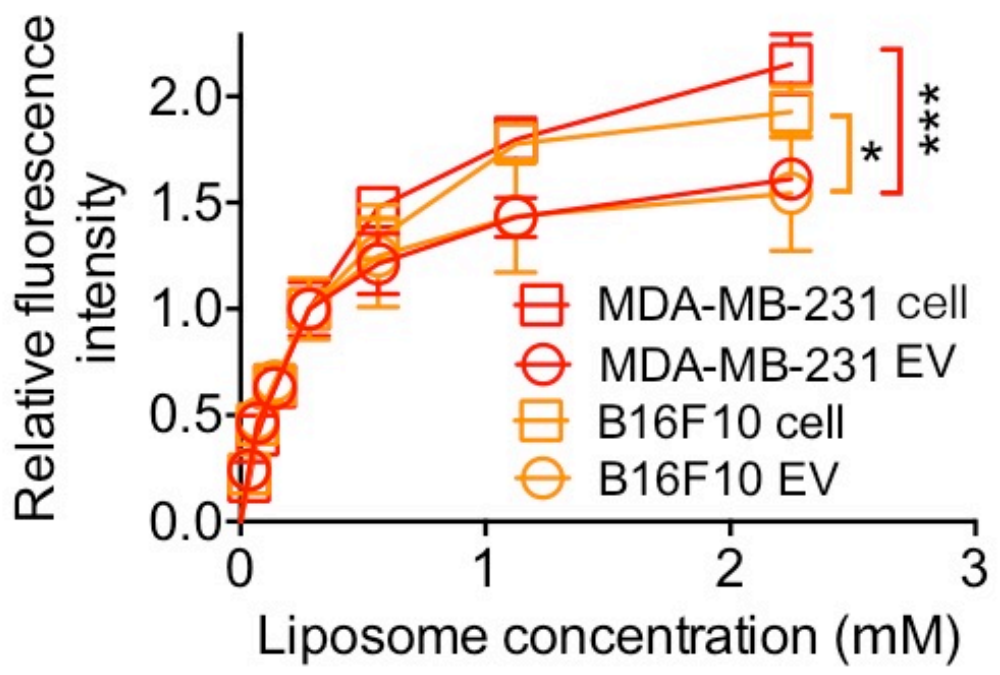

Figure S5 Fluorescence quantification of NBD-DPhPE lipids delivered to the cells and subsequently packaged into the EVs after MFL treatments at various concentrations $[\mathrm{n}=4 ; * P<$ 0.05 , and ${ }^{* * *} P<0.001$ by two-way ANOVA followed by Bonferroni post test.]. 

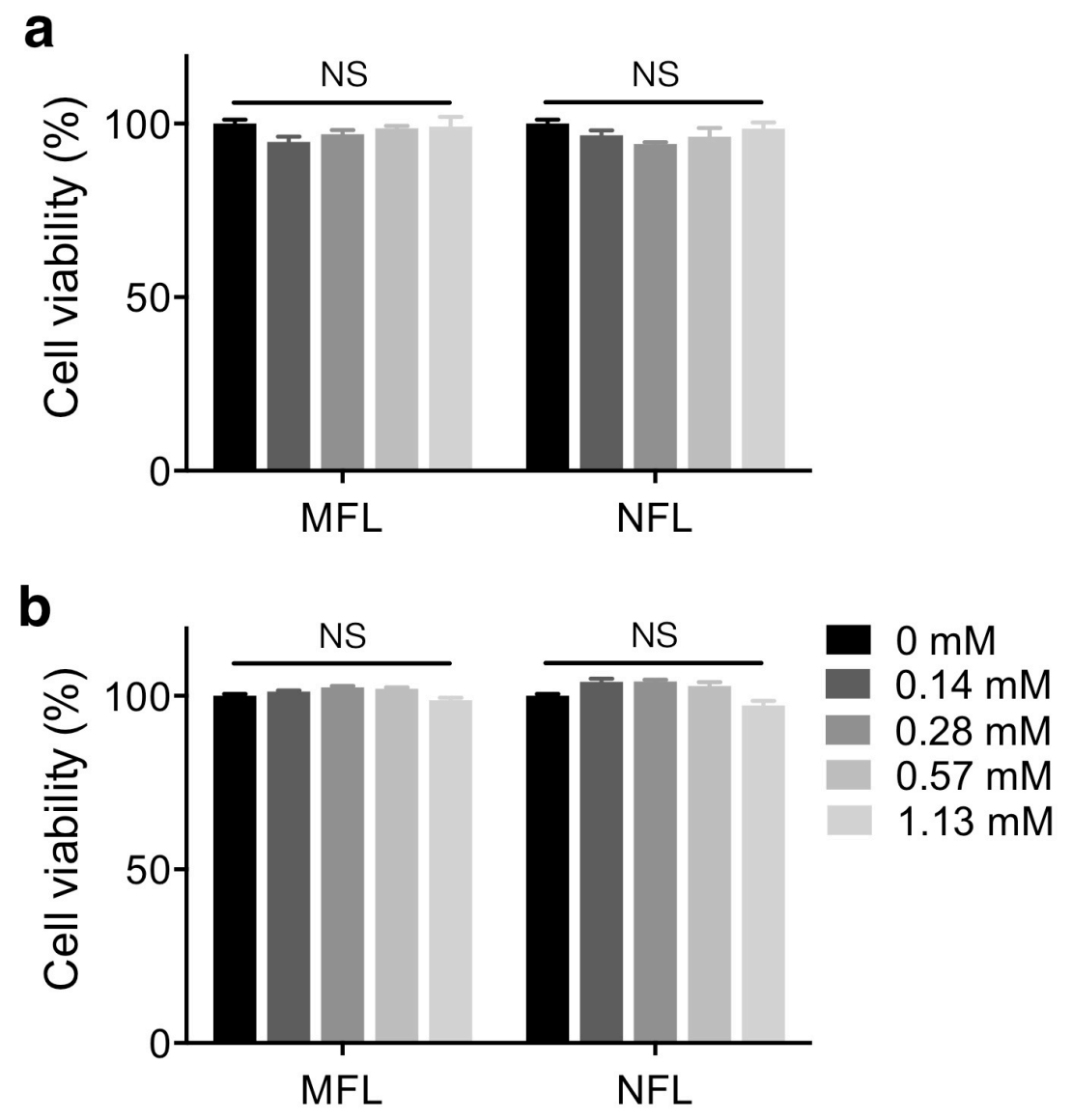

Figure S6 Cell viability of MDA-MB-231 (a) and B16F10 (b) after incubation with synthetic liposomes for 30 min at various lipid concentrations. Data represent averages \pm S.D. $[n=5$; NS, not significant by ANOVA] 


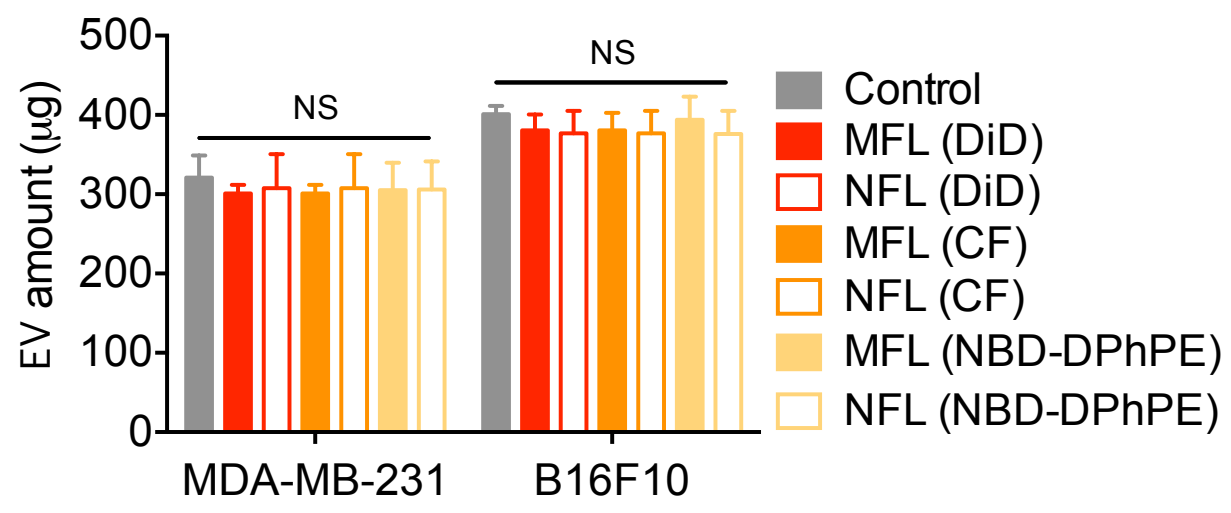

Figure S7 Amount of functional EVs produced from the cells treated with synthetic liposomes loaded with various functional agents. Data represent averages \pm S.D. $[n=5$; NS, not significant by ANOVA]. 
a

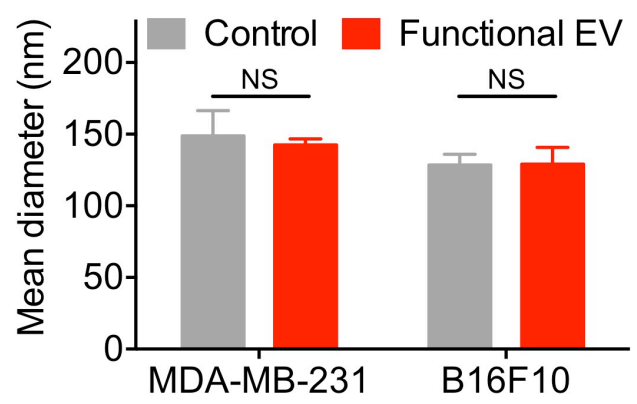

C
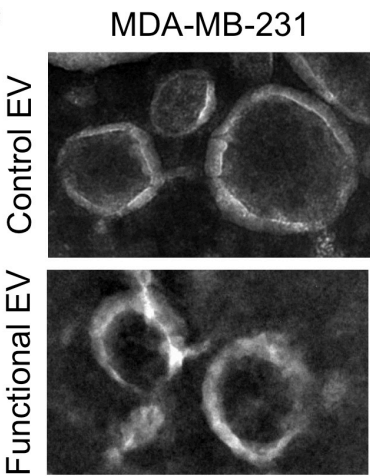

b

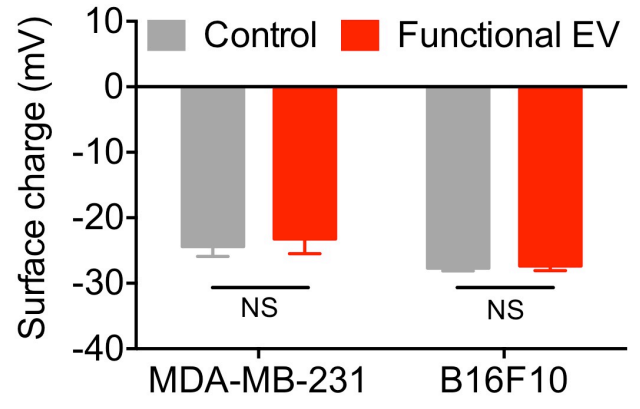

d

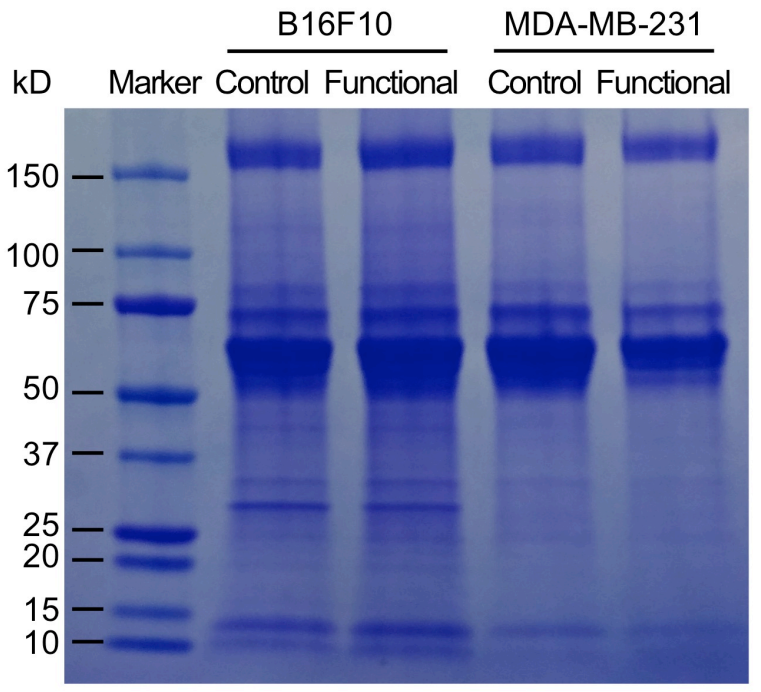

Figure S8 Physicochemical and biological properties of functional EVs produced by engineering parental cells with synthetic liposomes loaded with functional agents. (a-c) Transmission electron microscopic images (a), hydrodynamic sizes (b) and surface charges (c) of control EVs produced from untreated cells and functional EVs produced from MFL-treated cells. Scale bar indicates $50 \mathrm{~nm}$. (d) Coomassie blue staining of proteins associated with control EVs produced from untreated cells and functional EVs produced from MFL-treated cells. Data represent averages \pm S.D. [ $\mathrm{n}=3$; NS, not significant by ANOVA] 


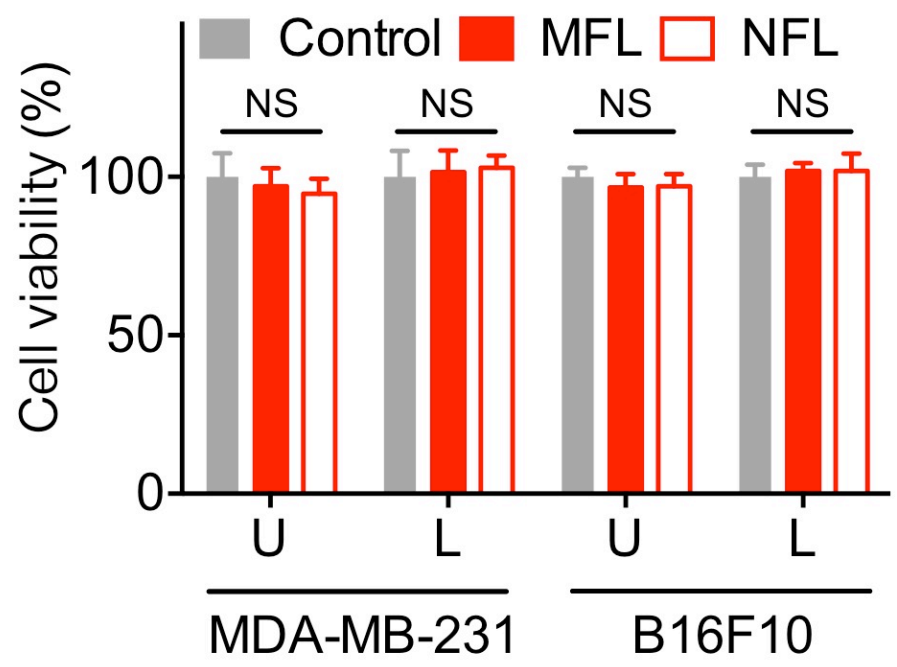

Figure S9 Therapeutic effects of tirapazamine(TPZ)-loaded EVs in the normoxic condition that are produced from the upper filter cells treated with TPZ-loaded liposomes. Data represent averages \pm S.D. $[n=5$; NS, not significant by ANOVA]. 

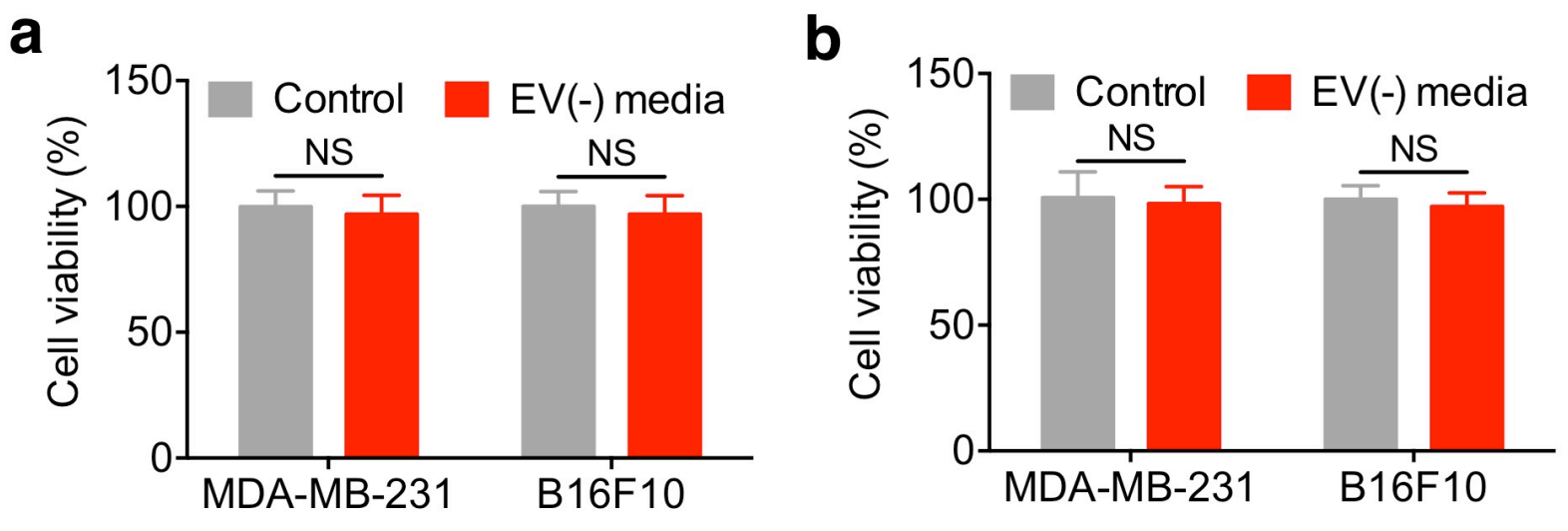

Figure S10 EV-mediated delivery of drugs. (a and b)Viability of cancer cells treated with the supernatant depleting the EVs loaded with the PTX (a) or TPZ (b). The cells were treated with drug-loaded MFLs for 30 min, washed to remove free MFLs in the media, and further incubated for $48 \mathrm{~h}$. The drug-loaded EVs were removed from supernatant of the treated cells by ultracentrifugation. Fresh cancer cells were incubated with the EV-depleted supernatant for $24 \mathrm{~h}$ (in hypoxic condition for TPZ), and the cell viability was evaluated using MTT assay. Data represent averages $\pm S . D[n=4 ; N S$, not significant by unpaired t-test $]$. 


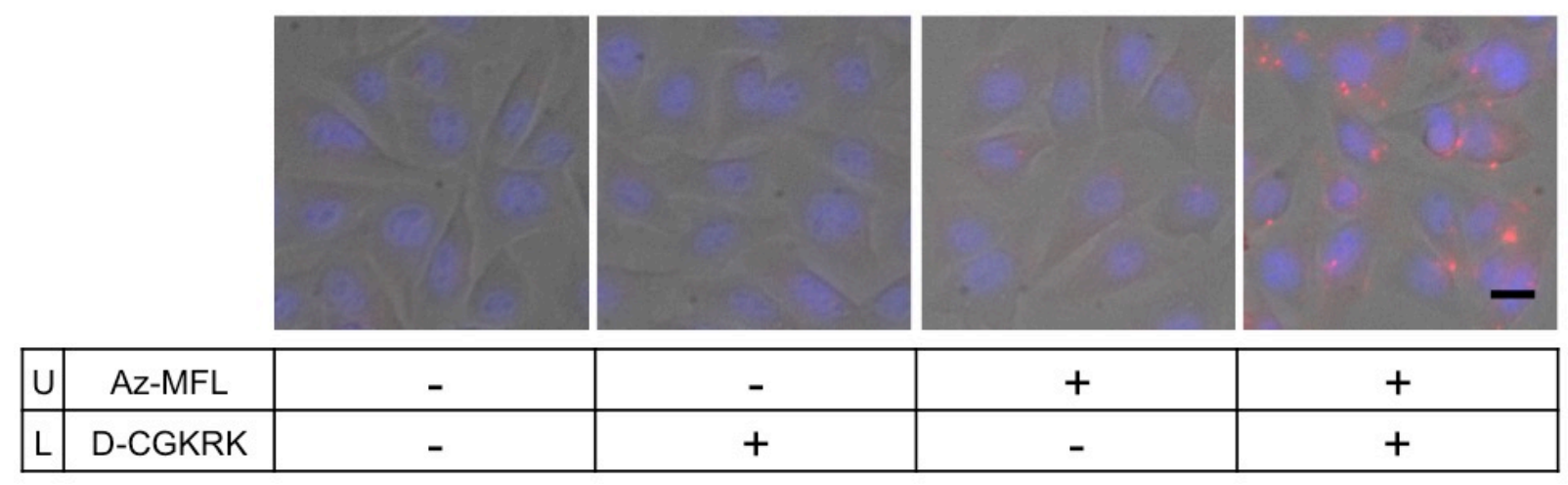

Figure S11 Fluorescent microscopic images of lower chamber (L) cells treated with EVs that were produced from the upper filter $(\mathrm{U})$ cells treated with various $\mathrm{DiD}$ (red)-loaded MFLs followed by in situ click conjugation with targeting peptide DBCO-CGKRK (D-CGKRK). Nuclei were stained with Hoechst (blue). Scale bar indicates $5 \mu \mathrm{m}$. 